\title{
Children's Reports of Disclosure Recipient Reactions in Forensic Interviews: Comparing the NICHD and MoGP Protocols
}

\author{
Elizabeth C. Ahern ${ }^{1}$ - Michael E. Lamb ${ }^{1}$
}

Published online: 12 July 2016

(C) The Author(s) 2016. This article is published with open access at Springerlink.com

\begin{abstract}
Reactions from confidantes who receive children's abuse disclosures can affect children's well-being and the likelihood that they will recant. Disclosure recipient (DR) reactions were coded in 95 forensic interviews of 4- to 13-year-old alleged sexual abuse victims. Half of the interviews were conducted using the National Institute of Child Health and Human Development (NICHD) Protocol (which includes a disclosure phase focused on the child's initial abuse report) and the other half using the Memorandum of Good Practice (MoGP), a predecessor of the Achieving Best Evidence (ABE) guidelines used in the UK today (which recommends asking about children's initial disclosures but has no designated disclosure phase). Children reported a variety of DR reactions, including supportive and unsupportive responses, and noted that many DRs questioned them about the allegations. NICHD interviews contained more references to DR reactions than MoGP interviews. NICHD interviews elicited more DR reaction information using invitations rather than more focused prompts and by asking children explicitly about their disclosures rather than relying on children to provide the information spontaneously. Findings indicated that children may be willing and able to provide disclosure information but may require prompting.
\end{abstract}

Keywords Disclosure $\cdot$ Children $\cdot$ Forensic interview

After children make initial disclosures of abuse, a series of discussions with children about the disclosure often ensues,

Elizabeth C. Ahern

eca35@cam.ac.uk

1 University of Cambridge, Cambridge, UK and this can include conversations with family members, friends, social service, and/or legal authorities (Stolzenberg and Lyon 2014). When cases are prosecuted, these conversations may be viewed as potential sources of contamination, so it may be especially important for children to be asked about the disclosure process early in the investigative process. In the present study, we compared the richness and spontaneity of children's reports of the disclosure process during forensic interviews conducted by officers trained to use the National Institute of Child Health and Human Development (NICHD) Protocol (Orbach et al. 2000) or the Memorandum of Good Practice (MoGP). The MoGP was the guidance provided by the British Home Office (1992) that evolved into the Achieving Best Evidence (ABE) guidance used today (Crown Prosecution Service and Ministry of Justice 2011).

\section{Why Is it Important to Ask About DR Reactions?}

Children's Health and Adjustment Others' reactions to children's disclosures of abuse are critical for several reasons. Caregivers' support and belief in disclosures of child maltreatment can affect children's well-being (Everson et al. 1989; Goodman et al. 1992; Gries et al. 2000) and have critical implications for children's placement post-disclosure (Leifer et al. 1993). Indeed, parental support may be a better predictor of psychological adjustment than abuse-related factors (e.g., Tremblay et al. 1999). In particular, disclosure recipients' (DR) support is positively associated with children's wellbeing whereas unsupportive or disbelieving reactions are associated with maladjustment (Tremblay et al. 1999). The kinds of caregiver reactions children receive (e.g., blame/anger, calm/support) have also been tied to children's feelings about their decisions to report abuse to caregivers (Hershkowitz et al. 2007). Children's anticipation of caregiver 
reactions may affect children's decisions to disclose and the likelihood that they may later recant (Malloy et al. 2011, 2013). Thus, it might be critical to ask children about others' reactions to their disclosures early in the investigation in order to facilitate appropriate interventions.

Legal Significance The context of children's initial disclosure can be controversial in court. Oftentimes, children's initial disclosures of abuse occur in brief conversations with caregivers (Lyon et al. 2012a). Caregivers may be represented as the source of children's reports (Bala et al. 2007; Jones \& McGraw 1987) when the suspects are estranged romantic partners of children's caregivers, and it may be alleged that the abuse disclosures were the result of coercive and suggestive questioning or the misinterpretation of ambiguous statements. In a recent study reviewing 72 criminal court transcripts of cases involving 6- to 16-year-old alleged victims of sexual abuse, all children were asked about their abuse disclosures, particularly about their initial reports to caregivers (Stolzenberg and Lyon 2014). Asking children about prior disclosures in forensic interviews may be especially important, as it is unlikely that adults coercing children to disclose abuse would instruct children on what to say about disclosure. To date, however, no researchers have examined the extent to which children are asked about their conversations with DRs in forensic interviews.

Defense attorneys are also likely to question children about initial disclosures in order to highlight changes in the children's reports before trial. Although these inconsistencies might reflect the natural limitations of memory and the effects of repeated interviews using many closedended questions (La Rooy et al. 2010), some inconsistencies might be explained by the level of support children received following disclosure (Malloy et al. 2007), particularly because DR support and belief influence children's willingness to disclose (Hershkowitz et al. 2007; Lawson and Chaffin 1992). A review of 257 substantiated cases of child sexual abuse involving 2- to 17-year-olds revealed that $20 \%$ of the children recanted abuse allegations; those children who were unsupported by nonoffending caregivers were especially at risk (Malloy et al. 2007). Information about children's initial abuse disclosures that are obtained in forensic interviews might help explain future inconsistencies as investigations progress. In addition, early identification of other DRs may later be useful if it becomes necessary to corroborate details about the disclosed incident. For example, in a study of 30 7- to 12-year-old suspected victims, children's reports of their initial disclosures to parents in forensic interviews often mirrored the parents' reports (Hershkowitz et al. 2007).

\section{Importance of Asking About Disclosure in Forensic Interviews}

It is important to document disclosure processes early in the investigation. The court environment is a poor venue in which to inquire about children's disclosure history for several reasons, including substantial delays between children's disclosures and their trial testimony (Goodman et al. 1992), and the fact that defense and prosecuting attorneys have contrasting views about the allegation, and often ask closed-ended questions that can be suggestive and difficult for child witnesses to understand (e.g., Zajac et al. 2012). Thus, children are better positioned to describe the disclosure process when questioned by trained and unbiased forensic interviewers soon after the initial disclosure has been made, especially when recordings of the children's forensic interviews may be played in court.

\section{Child Forensic Interview Studies Examining Children's Prior Disclosures}

Few researchers have examined children's descriptions within forensic interviews of what happened during the disclosure process. Most studies have focused on the anticipated or actual consequences of disclosure (Hershkowitz et al. 2007; Malloy et al. 2011) and reasons for disclosure (Malloy et al. 2013) rather than on how children report that DRs actually reacted or the ways in which disclosure information was elicited. Children might not be aware that forensic interviewers want to know whom they told, what was said, how they felt about disclosing, and how others reacted (Ahern and Lyon 2011). Thus, children may need to be prompted by interviewers to talk about the disclosure process, perhaps because they expect to focus exclusively on the abuse and not on the disclosure. Studies that have examined children's reports of DRs in forensic interviews (Hershkowitz et al. 2007; Malloy et al. 2011, 2013) have focused on interviews conducted using the NICHD Protocol which includes a disclosure phase. However, many interviewers around the world do not use the NICHD Protocol. It is possible that interviewers not using the NICHD Protocol may not question children about the disclosure process or rely on less specific guidance. For example, interviewers may focus on children's allegations to the exclusion of the disclosure process or, perhaps, only ask "whom" the child reported to without additional inquiries into the ways in which confidantes responded to their disclosure.

The structured NICHD Protocol was developed on the basis of decades of developmental research on memory and communication (e.g., see Lamb et al. 2007, for more details). After children have exhaustively described their experiences of abuse, they are encouraged to describe what had happened since those incidents. When children do not describe the disclosure process in detail spontaneously, they 
are asked additional questions to determine how others discovered the allegation, who they were, and what happened during each interaction. In contrast, the Memorandum of Good Practice on Video-Recorded Interviews With Child Witnesses for Criminal Proceedings (Memorandum of Good Practice (MoGP); Home Office 1992) offered less guidance about how to inquire about children's disclosure histories and may be representative of other non-NICHD interviews in which general guidance (without specific examples) is offered. The MoGP permitted interviewers to ask about DRs in order to help explain why the children were being interviewed (Home Office, 1992, 3.7) and also encouraged interviewers to ask children if they had tried to tell anyone else about the abuse before (Home Office, 1992, 3.29) but did not suggest prompts that might be used (e.g., "How did people find out about this?"). The MoGP's successor, the $\mathrm{ABE}$ guidance, recommends asking possible victims about adult or peer support, whether the child has been able to tell others about the abuse, and parental reactions to such disclosures but does not specifically indicate how these inquiries might be phrased. In both the NICHD and MoGP guidelines, interviewers are advised to ask open-ended questions before resorting to more closed-ended questions and never to ask suggestive questions.

\section{Current Study}

In the present study, we examined children's reports of DR reactions in 95 forensic interviews of 4- to 13-year-old children alleging sexual abuse. Half of the interviews were conducted using the NICHD Protocol (which includes a disclosure phase) and the other half were conducted by officers trained to use the MoGP (which did not include a disclosure phase but offered general recommendations). We wanted to use the MoGP interviews as examples of interviews, conducted using less structured guidance than the NICHD Protocol, in which interviewers may be aware of best practices but fail to change their behavior accordingly (Lamb et al. 2009).

The study was designed to address the following questions: Did children identify to whom they disclosed abuse? Did disclosure information need to be requested explicitly? Was disclosure information sought using open-ended prompts? What DR reactions did children describe?

We hypothesized that, in comparison with MoGP interviewers, NICHD interviewers would do the following:

a) Elicit more references to DRs and more DR reactions than MoGP interviewers because the NICHD Protocol structure specifically illustrates how children should be asked about the disclosure process (Hershkowitz et al. 2007)

b) Elicit more DR information from open-ended prompts than MoGP interviewers because NICHD interviewers tend to rely on invitations more than MoGP interviewers (Lamb et al. 2009)

c) Elicit DR information using questions that reference disclosure content specifically (e.g., "How did people find out about this?") because NICHD interviewers are encouraged to use such prompts when the information has not been provided

\section{Method}

In this study, we examined 95 forensic interviews of alleged sexual abuse victims by police officers in a mid-sized constabulary in the British midlands (described by Lamb et al. 2009). All were the first evidentiary interviews of these children, conducted by the police in accordance with the MoGP (Home Office, 1992). The 20 boys and 75 girls interviewed averaged 9.3 ( $\mathrm{SD}=2.57$ ) years of age (range 48-157 months). Forty nine of the interviews were conducted between 1999 and 2001 by five police officers following the NICHD Protocol, whereas a matched sample of 46 interviews was conducted by the same interviewers or one of ten colleagues (to supplement the required number of matched interviews) in the same constabulary immediately before the protocol was implemented. Most of the interviewers had limited experience (less than 1 year) investigating sex crimes involving children (rather than adults) before the study began, and interviews by more experienced investigators were excluded from both the protocol and MoGP groups. Interviewers in the protocol condition had been trained to use the NICHD Investigative Interview Protocol (Orbach et al. 2000; Sternberg et al. 2001), whereas interviewers in the comparison condition had been trained to follow the MoGP as required by the Home Office (Davies et al. 1998). Interviews in the comparison group were individually matched with protocol interviews as closely as possible with respect to age (within 12 months), the severity or type of alleged abuse (exposure (protocol, $n=2$, MoGP, $n=3$ ); fondling over (protocol, $n=10$, MoGP, $n=11$ ) or under (protocol, $n=15$, c MoGP, $n=10$ ) clothes, penetration (protocol, $n=12$, MoGP, $n=13$ )), the relationship between alleged victim and perpetrator (immediate family members (protocol, $n=12$, MoGP, $n=13$ ), other family (protocol, $n=15$, MoGP, $n=10$ ), familiar (protocol, $n=21$, MoGP, $n=22$ ) and unfamiliar (protocol, $n=1$, MoGP, $n=1)$ ], and whether or not the abuse had occurred once ( $n=10$ and $n=17$ in MoGP and protocol conditions, respectively) or multiple times ( $n=36$ and $n=32$ in MoGP and protocol conditions, respectively). For purposes of some analyses, the children were divided into two age groups, 4-8year olds $(n=34)$ and $9-13$-year olds $(n=61)$. We chose to match with respect to age of interviewee, relationship to perpetrator, and both severity and frequency of abuse in light of 
extensive evidence from previous research showing that these factors strongly and reliably affect the likelihood that children will be informative when interviewed (Hershkowitz et al. 2006).

All the interviews were transcribed, checked for accuracy, and checked to ensure that all personal identifiers were deleted before transcripts were sent to the researchers.

\section{Interviewer Training}

Prior to the implementation of the structured NICHD Protocol, all interviewers participated in an intensive 5-day training program during which the conceptual and empirical support for all phases of the interview were explained and videotapes illustrating both appropriate and inappropriate interview techniques were shown. After familiarizing themselves with the structured protocol, interviewers practiced using the protocol to interview role-playing confederates who based their responses on real cases. Interviewers were then observed conducting field forensic interviews using the protocol and were given feedback on their practices and techniques. Written feedback was provided on all transcribed field interviews until the study ended. In addition, individual meetings with the research team every 6-8 weeks focused on critical analysis of the interviewers' adherence to the protocol and its strategies in their recent interviews.

Although all interviewers had previously been trained to conduct MoGP interviews, there had been no uniform policy regarding the training of police officers to follow the MoGP and to implement the superior practices endorsed by it. Almost all training was offered jointly to police officers and social workers by the local Area Child Protection Committee through participation in a 5-day investigative interviewing course (Davies et al. 1998).

\section{Coding Procedure}

Two research assistants identified occasions when a DR was referenced and who the DR was. When DR reactions were mentioned, the preceding prompt was coded for content (whether the prompt explicitly asked for disclosure information) and format (invitation, directive, forced choice, or suggestive utterance type). The types of DR reactions that children described (if any) were also examined. Every transcript was reviewed twice, once by each coder. Research assistants and the first author met throughout the process of data extraction and coding.

DRs DRs were classified as parents (mother, father, step-parents), familiar adults (aunt, uncle, peer's parent), persons of authority (social workers, police, teachers), and peers/siblings.
Prompts Eliciting DR Reactions In order to assess how children reported disclosure information, prompts that elicited the first DR reactions reported by each child were coded for question content and format.

Question Content 1. Did not ask about disclosure directly (no disclosure content). Most often, prompts that did not ask for disclosure-related information asked about the allegation ("Tell me why you came to talk to me today," "What happened in the bed?", "You said he touched you, tell me more about him touching you?", "What happened next?"). Thus, children providing DR reaction information to no-disclosure-content questions did so spontaneously without explicit prompting by the interviewers.

2. Asked child for the identity of whom she/he told (DR identity). These prompts asked for the identities of the DRs ("Did you tell your mum?", "Who was the first person you told?").

3. Asked about other aspects of the disclosure beyond the identity of the DR (disclosure process). These prompts focused broadly on the context of the disclosure, which often included the process by which others discovered the child's abuse ("How did DR find out?"), what occurred during the disclosure ("What happened when you told DR?"), inquiries about the children's statements ("What did you say?"), and how DRs responded ("What did she/ he do/say?", "Was it ok telling her?").

Question Format Question format categories included NICHD utterance types as defined in other reports (e.g., Lamb et al. 2009):

1. Invitations. Input-free utterances, including questions, statements, or imperatives prompting free-recall responses from the child. Such utterances do not delimit the child's focus except in a general way ("Tell me everything that happened") or use details disclosed by the child as cues ("You mentioned that he touched you. Tell me everything about the touching").

2. Directives. These refocus the child's attention on details or aspects of the alleged incident that the child has already mentioned, providing a category for requesting additional information using "Wh-" questions (cued recall). For example, the interviewer might say, "What did you say?" when the child mentioned that she had disclosed.

3. Option-posing utterances. These focus the child's attention on details or aspects of the alleged incident that the 
child has not previously mentioned, asking the child to affirm, negate, or select an investigator-given option, thus using recognition memory processes, but do not imply that a particular response is expected. For example, the investigator might ask "Did you tell your mother?" when the child mentioned she told someone.

4. Suggestive utterances. These are stated in such a way that the interviewer strongly communicates what response is expected ("He forced you to do that, didn't he?") or they assume details that have not been revealed by the child (child: "We laid on the sofa." Interviewer: "He laid on you or you laid on him?"). None of the questions coded for this study were suggestive in nature perhaps due to our focus on disclosure specifically.

DR Reactions DR reactions were also classified into naturally occurring categories. DR reactions included (a) reporting the disclosure to others (e.g., to the child's family or to the police), (b) expressing empathy or compassion (e.g., hugging the child, saying "I'm sorry", offering reassurances), (c) encouraging the child to disclose to an adult (e.g., usually to the child's parents), (d) questioning the child about the abuse, (e) reacting strongly (e.g., crying, yelling, getting "upset", confronting the suspect), and (f) being unsupportive (e.g., disbelief, recommending the child to stay silent).

Given our broad interest in DR reactions, reactions included those that the child experienced directly (child told parent, parent expressed disbelief (parent reaction $=$ disbelief)), observed when another person disclosed on the child's behalf (child watched cousin tell aunt and aunt reacted in disbelief (peer reaction $=$ reporting the disclosure to others; familiar adult reaction = unsupportive)), and through hearsay (child was told by friend that friend told her grandma and her grandma disbelieved disclosure (peer reaction $=$ reporting the disclosure to others; familiar adult reaction $=$ unsupportive)).

Twenty percent of the transcripts were independently coded for content, format, and DR reaction by one of the authors and a research assistant; the inter-rater agreement coefficients (Kappas) were .77, .89, and .73, respectively.

\section{Results}

Preliminary analyses revealed no effects due to gender, abuse severity, and suspect relationship on whether DRs or DR reactions were mentioned by children. Effects due to age are reported where applicable.

First, we examined the identity of DRs: parents (mentioned by $72 \%$ of the children), familiar adults (37\%), siblings/peers (46\%), and persons of authority (25\%). The percentages sum to more than 100 because some children disclosed to more than one person. Of the 95 children, $88 \%(n=84)$ reported disclosures $(M=1.80, \mathrm{SD}=1.10$; range $0-4)$. Nearly three quarters $(72 \% ; n=68)$ of the children reported DR reactions $(M=3.76, \mathrm{SD}=4.39$; range $0-18)$.

Next, we examined the average number of DRs and DR reactions each child mentioned using univariate analyses of variance (ANOVA). Age (4 to 8 years, 9 to 13 years) and procedure (NICHD, MoGP) were entered as between subject factors.

For the number of DRs children mentioned, there were main effects due to age, $F(1,91)=11.01, p=.001$, $\eta_{p}{ }^{2}=.11$, and procedure, $F(1,91)=8.37, p=.005$, $\eta_{p}{ }^{2}=.08$. Older children reported $(M=2.05, \mathrm{SD}=1.15)$ more DRs than younger children $(M=1.35, \mathrm{SD}=.85)$. More DRs were mentioned in the NICHD $(M=2.14, \mathrm{SD}=1.00)$ than the $\operatorname{MoGP}(M=1.43, \mathrm{SD}=1.09)$ interviews.

For the number of DR reactions children mentioned, there were main effects for age, $F(1,91)=26.21, p<.001$, $\eta_{p}{ }^{2}=.22$, and procedure, $F(1,91)=13.35, p<.001$, $\eta_{p}{ }^{2}=.13$, as well as an age $\times$ procedure interaction, $F(1$, $91)=10.40, p=.002, \eta_{p}{ }^{2}=.10$. Older children reported $(M=5.13, S D=4.84)$ more DR reactions than younger children $(M=1.29$, SD $=1.64)$. More DR reactions were mentioned in the NICHD $(M=5.38, \mathrm{SD}=5.12)$ than the MoGP $(M=2.04, \mathrm{SD}=2.56)$ interviews. The age $\times$ procedure interaction revealed that, for the older children, the NICHD procedure $(M=7.64, \mathrm{SD}=5.08)$ elicited more $\mathrm{DR}$ reactions than the MoGP $(M=2.53, \mathrm{SD}=2.84), t(59)=4.83, p<.001$, whereas both procedures elicited similar (lower) numbers of DR reactions from younger children (NICHD: $M=1.44$, $\mathrm{SD}=1.65$; MoGP: $M=1.13, \mathrm{SD}=1.67)$.

Of primary interest was whether children's reports of DRs or DR reactions varied depending on interviewing procedure for children of different ages. The dichotomous scores (mentioned, not mentioned) for reporting DRs and DR reactions were thus entered into separate logistic regressions with age (4 to 8 years, 9 to 13 years) and procedure (NICHD, MoGP) entered as predictors.

For reporting a DR, although the model was significant, $X^{2}$ $(2, N=95)=18.21, p<.001$, no predictor had a significant effect. However, every NICHD interview contained at least one reference to a DR $(n=45)$ whereas only $76 \%$ of the MoGP interviews did $(N=35), \chi^{2}(1,95)=13.25, p<.001$, showing that children were 1.32 times more likely to report a DR in NICHD than MoGP interviews.

For reporting a DR reaction, the model was significant, $X^{2}$ $(2, N=95)=15.22, p<.001$, with age and procedure emerging as significant predictors. Children in the NICHD (86\%, $n=42$ ) condition were more likely to report DR reactions than children in the MoGP group (57 \%,n=26), Wald =9.74, $p=.002, \mathrm{OR}=1.53$. Older children $(79 \%, n=48)$ were more likely to report DR reactions than younger children (59\%, $n=20$ ), Wald $=4.84, p=.03, \mathrm{OR}=1.34$. 


\section{Did Children Report DR Reactions Spontaneously?}

To assess the spontaneity with which children reported DR reactions, we limited the following analyses to the first mention of a DR reaction in each interview. Spontaneity was assessed using the question content codes. Table 1 displays the percentage and frequency of cases reporting DR reactions in relation to question content and format.

A chi-square test comparing protocol and question content was significant, $\chi^{2}(2,68)=9.63, p<.001$. Examination of the percentages revealed that the MoGP interviewers elicited proportionally more DR-reaction information using nodisclosure-content and DR-identity questions than NICHD interviewers. In contrast, the NICHD interviewers elicited proportionally more DR-reaction information using explicit prompts about disclosure.

\section{What Types of Question Format Elicited DR Reactions?}

A multinomial regression was conducted to examine the effect of the protocol (NICHD, MoGP) on question format (invitation, directive, option posing). Because NICHD protocol interviews contained proportionally more open-ended prompts than MoGP interviews overall (Lamb et al. 2009), the percentages of directives, invitations, and option-posing prompts employed in the average child interview of that type were entered as covariates. The final model predicted the dependent variable over and above the intercept-only model, $\chi^{2}$ $(8)=15.27, p=.05$. Examination of the percentages revealed that the NICHD interviewers elicited more of children's initial reports of DR reactions using invitations than the MoGP interviewers, and that the MoGP interviewers used more optionposing utterances than NICHD interviewers to elicit DR reactions (Table 1).

\section{What Did DRs Reportedly Do After Disclosure?}

The kinds of DR reactions children reported were explored. Table 2 shows the percentage and number of children reporting DR reactions during their interviews. Half of the children mentioned that the DR told another person about their disclosure. A third of the children reported that the DR reacted with strong emotions (e.g., crying, threatening the suspect), questioned or talked to the child about what happened, or were unsupportive (e.g., ignored the child's disclosure). Twentyfour percent of the children reported receiving empathic responses and $15 \%$ reported that the DRs encouraged them to disclose the abuse to adults.

Children's utterances indicating that DRs spoke to them about the abuse were further explored. Out of the 43 such instances, $58 \%$ included verbatim quotes of what the DR stated (e.g., "Did he?", "How far did he go?"), $28 \%$ were generic reports that a DR "asked" about the incident or "asked me questions" without specifying what the questions were, and $14 \%$ were generic reports that a DR "talked to me" about the incident.

\section{Discussion}

Because DR reactions have important implications for children's well-being and case prosecution, the purpose of the present study was to examine to whom children reported disclosing, the kinds of questions that elicited DR reactions, and the types of DR reactions children mentioned in forensic interviews. A particular focus was on differences between interviews conducted using the NICHD and MoGP protocols. Both protocols encourage use of open-ended prompts before employing more closed-ended prompts and suggest asking about children's disclosure processes, with the NICHD Protocol providing explicit examples of recommended prompts. Compared to the MoGP interviews, we expected NICHD interviews to contain more references to DRs and DR reactions, and that most DR information would be elicited using open-ended questions that ask for disclosure information explicitly. We anticipated that a variety of DR reactions would be reported.
Table 1 Percentage

(frequencies) of question content and format eliciting first DR reaction by procedure

\begin{tabular}{lll}
\hline & \multicolumn{2}{l}{ Procedure } \\
\cline { 2 - 3 } & $\%(n)$ Cases within NICHD & $\%(n)$ Cases within MoGP \\
\hline Question Content & $36 \%(14)$ & \\
Did not ask about disclosure & $8 \%(3)$ & $55 \%(12)$ \\
Asked for DR identity & $56 \%(22)$ & $27 \%(6)$ \\
Asked about disclosure process & $50 \%(21)$ & $18 \%(4)$ \\
Question Format & $41 \%(17)$ & $23 \%(6)$ \\
Invitation & $10 \%(4)$ & $43 \%(13)$ \\
Directive & $27 \%(7)$ \\
Option posing &
\end{tabular}


Table 2 Percentages (frequencies) of children reporting kinds of reactions

\begin{tabular}{lc}
\hline Disclosure recipient reactions & \% Cases $(n)$ \\
\hline Reported the disclosure to others (e.g., told a peer, familiar adult, phoned the authorities) & $50 \%(48)$ \\
Strong reaction (cried, confronted the suspect) & $36 \%(34)$ \\
Questioned the child (e.g., "Mum talked to me about what happened.", "How far did he go?") & $34 \%(32)$ \\
Unsupportive (disbelief, anger at child) & $32 \%(30)$ \\
Expressed empathy (e.g., hugging the child, "Are you ok?", "I'm so sorry") & $24 \%(23)$ \\
Encouraged child to disclose to adult (e.g., "You have to tell your Mum.") & $15 \%(14)$ \\
\hline
\end{tabular}

Percentages sum to greater than $100 \%$ because some children reported multiple DR reactions

\section{Protocol Effects}

Our hypotheses regarding protocol were largely supported. Children in the NICHD interviews were more likely to report whom they disclosed to in addition to the ways in which the DRs reacted to their disclosures than were children in MoGP interviews. As we expected, a large percentage of NICHD DR reactions were made in response to explicit questioning about the disclosure by interviewers using open-ended questions, which occurred after children's accounts of the allegations were complete.

The fact that proportionally more children in the NICHD interviews reported DR reactions, largely in response to disclosure process prompts, suggests that interviewers may often need to ask for this information explicitly although some children in both conditions reported such information spontaneously. Previous reports examining children's DR utterances in relation to the anticipated consequences of (Malloy et al. 2011) and reasons for disclosing (Malloy et al. 2013) also reported that many children required explicit prompting before such information was disclosed (23 to $70 \%$ of children).

In the NICHD interviews, DR-reaction information was elicited more often in response to open-ended prompts than in MoGP interviews, a finding consistent with other research showing that interviewers using the NICHD Protocol use more open-ended prompts than those using the MoGP (Lamb et al. 2009). This may be because the NICHD Protocol includes examples and question stems (e.g., "Tell me more about $\left.X^{\prime \prime}\right)$ rather than only general guidelines. In addition, unlike the NICHD interviewers, the MoGP interviewers were not routinely provided with on-going supervision regarding interview quality. These findings illustrate the importance of continual training and specifically questioning children about their disclosure histories using prepared questions (e.g., "How did people find out about this?").

Finally, the NICHD procedure elicited more DR reactions than the MoGP from older children, whereas both procedures elicited similar numbers of DR reactions from the younger children. It is possible that younger children required more explicit questions about DR reactions before reporting that kind of information (e.g., "What did your mom do after you told her?"). Older children may find it easier to provide DR information in response to open-ended questions about the disclosure process.

\section{DR Reactions Reported}

Various kinds of DR reactions were reported, including some that might produce a sense of comfort (e.g., empathy) and others that might foster unease (e.g., confronting the suspect, crying in response to the disclosure). A third of the children reported that a DR reacted to their disclosure by talking or questioning them about the incident. In many of these reports, children generically referred to the fact that someone talked to them or questioned them about the incident. Fewer children reported some of the actual dialogue that took place between themselves and the DR. Some DR questions appeared to stem from initial shock ("Did he?") with others seemingly designed to understand abuse severity ("How far did he go?"). None of the conversational exchanges appeared very coercive, although these dialogs were often not explored exhaustively by the interviewers. Questioning indicated that the DRs were trying to understand the nature of the abuse and to determine whether formal reporting was warranted. Interviewers might want to ask children to elaborate more on what children and DRs said in order to forestall later allegations that the interactions were suggestive (California v Ortega), even though they may doubt the verbatim accuracy of the reported dialogues (Lawson and London 2015).

The present study highlights the need for forensic interviewers to ask children explicitly about the disclosure process if they do not volunteer the information on their own. Certainly, other research shows that children must sometimes be prompted for information using open-ended prompts when the information is not spontaneously reported (e.g., in the case of emotional content; Lyon et al. 2012b). From this examination of forensic interviews, it appears that children may be capable of describing their disclosures, usually demonstrating the non-coercive nature of children's initial reports to caregivers. However, our sample was limited to suspected victims of sexual abuse. It is possible that asking children about their initial disclosures in hotly contested custody cases, where there may be higher levels of coercion, might yield less accurate reports. 
It is unclear what aspects of the abuse or disclosure process might make some children more likely to provide DR information spontaneously. For example, it is possible that children receiving unsupportive responses might report the DR reactions more often than those who receive supportive reactions because the children feel concern, guilt, or anger about the DRs' reactions. On the other hand, children who receive such unsupportive responses might feel more uncomfortable reporting the information altogether. Such questions would provide valuable information about children's general sense of comfort when disclosing difficult events.

Interviewers managing children's reluctance in forensic interviews might be able to review children's previous reports to DRs and ask children about their feelings at the time of previous disclosures. For example, when children received support during their initial disclosures, they might be reminded of that positive reaction during the forensic interview. When children were not supported during their initial disclosure, the interviewer may choose to empathize and recognize that experience. Moreover, asking children about DR reactions can help interviewers understand why children might be reluctant and thus to offer more appropriate reassurances.

Some might argue that asking children about the disclosure process is secondary to investigation of the alleged crime and unnecessarily prolongs the interview. Although the central purpose of the forensic interview is to focus on the alleged events, evaluation of the informant's veracity can be aided by eliciting as much information as possible about not only the charge itself but the surrounding circumstances and context. Explorations of the disclosure process can also provide investigative leads regarding other witnesses to contact.

If it becomes apparent that children need a break from being interviewed, a follow-up interview can be conducted to focus on the disclosure process. Nonetheless, NICHD interviews have been including the disclosure phase for decades, and, as shown in this sample, children as young as four years of age were able to respond with disclosure-related information after also describing the incidents under investigation.

Lastly, it may be valuable to examine the extent to which children report both why they disclosed and why they delayed disclosure (Ahern and Lyon 2011). The fact that such motivations might be hotly debated in court makes it all the more important to offer children an opportunity to report this information in the initial forensic interviews which can be presented as evidence in court. Moreover, the more information interviewers can elicit about the context of abuse and children's disclosures, the more information available to juries trying to decide whether or not a certain incident occurred, especially when children's reports might become inconsistent over time in response to external pressures (Malloy et al. 2007). Finally, beyond its effects on the pursuit of justice, information about DR reactions should be sought in investigative interviews because they can affect children's well-being (Goodman et al. 1992).

\section{Limitations}

Several limitations need to be acknowledged. The vast majority of children's reports about DRs and their reactions were based on conversations the children had. To date, there has been very little research on individuals' recall of conversations, let alone on research with children's memory for prior conversations in legal contexts (Davis and Friedman 2007). Although source monitoring limitations may make it difficult for children to recall accurately such details as what each person stated or how conversations began, children may be able to remember the gist of conversations and whether the DRs were being supportive or not. Additionally, not only did the NICHD and MoGP guidance differ, but the amount of supervisions and level of training varied as well, with the NICHD Protocol offering interviewers more structured and systematic guidance. Because the NICHD interviews were conducted shortly after the MoGP interviews, some of the differences between protocols may be attributed to interviewer experience. However, as some studies suggest - interviewer experience does not necessarily lead to improved skills (Powell et al. 2008).

DR thoughts and reactions may vary across time and situation (Elliott and Carnes 2001), suggesting that caregivers' initial reactions may not predict their ability to believe, support, and protect children in the future (Salt et al. 1990). Nonetheless, our study examined caregivers' initial responses because they occur when children may be most uncertain about disclosing and thus most likely to retract allegations.

Although it is possible that children might not remember conversations accurately, it may be critical to understand how children recall their earlier disclosures. Their memories of disclosure interactions, even if somewhat inaccurate, likely have significant implications for their psychological adjustment.

Although our sample largely comprised interviews from the late 1990s, similar results would likely be obtained if present day NICHD Protocol interviews were compared with those conducted by less intensely monitored non-NICHD interviewers, who may well be aware of best interviewing practice but fail to change their behavior accordingly. A recent Norwegian study documented that, despite extensive investment in the training of forensic interviewers across the country, neither the use of undesirable practices (e.g., suggestive prompts) nor the use of recommended practices (e.g., openended questions) changed over a 22-year period (19902012) (Johnson et al. 2015). Thus, it is possible (albeit unfortunate) that present day interviews may resemble those we studied.

\section{Future Directions}

Children's reports about their disclosure history help investigators understand both the circumstances of the abuse and how the 
children's lives might have changed following disclosure. This kind of information might be especially important to examine in juror perception studies. Specifically, researchers might test how children's reports of their disclosure influence their perceived credibility in the courtroom. Moreover, it is possible that DR reactions may be associated with indicia of reluctance, with children experiencing negative reactions being less comfortable making disclosures during forensic interviews.

Acknowledgments This research was supported by the Nuffield and Jacobs Foundations. We thank Emma Douma, Juman Hamza, and Misun Yi who helped with data extraction and coding.

Open Access This article is distributed under the terms of the Creative Commons Attribution 4.0 International License (http:// creativecommons.org/licenses/by/4.0/), which permits unrestricted use, distribution, and reproduction in any medium, provided you give appropriate credit to the original author(s) and the source, provide a link to the Creative Commons license, and indicate if changes were made.

\section{References}

Ahern EC, Lyon, TD (2011) Supplemental investigative interview questions. Retrieved from: https://works.bepress.com/thomaslyon/74/

Bala N, Mitnick M, Trocme N, Houston C (2007) Sexual abuse allegations and parental separation: smokescreen or fire? J Fam Stud 13:26-56

Crown Prosecution Service \& Ministry of Justice (2011) Achieving best evidence in criminal proceedings. http://www.cps.gov. $\mathrm{uk} /$ publications/docs/best_evidence_in_criminal_proceedings.pdf

Davies G, Marshall E, \& Robertson N (1998) Child abuse: training investigative officers (police research series, No. 94). London: Home Office Police and Reducing Crime

Davis D, Friedman RD (2007) Memory for conversation: the orphan child of witness memory researchers. In: Toglia MP, Read JD, Ross DF, Lindsay RCL (eds) Handbook of eyewitness psychology, Memory for events, vol 2. Erlbaum, Mahwah, pp. 3-57

Elliott AN, Carnes CN (2001) Reactions of nonoffending parents to the sexual abuse of their child: a review of the literature. Child Maltreat 6:314-331. doi:10.1177/1077559501006004005

Everson MD, Hunter WM, Runyon DK, Edelson GA, Coulter ML (1989) Maternal support following disclosure of incest. Am J Orthopsychiatry 59:197-207

Gries LT, Goh DS, Andrews MB, Gilbert J, Praver F, Naierman-Stelzer D (2000) Positive reactions to disclosure and recovery from child sexual abuse. J Child Sex Abus 9:29-51

Goodman GS, Taub EP, Jones DP, England P. et al. (1992) Testifying in criminal court: emotional effects on child sexual assault victims. Monograph of the Society for Research on Child Development

Hershkowitz I, Orbach Y, Lamb ME, Sternberg KJ, Horowitz D (2006) Dynamics of forensic interviews with suspected abuse victims who do not disclose abuse. Child Abuse Negl 30(7):753-769. doi:10.1016/j.chiabu.2005.10.016

Hershkowitz I, Lanes O, Lamb ME (2007) Exploring the disclosure of child sexual abuse with alleged victims and their parents. Child Abuse Negl 23:175-190

Johnson M, Magnussen S, Thoresen C, Lønnum K, Burrell LV, Melinder A (2015) Best practice recommendations still fail to result in action: a national 10-year follow-up study of investigative interviews in CSA cases. Appl Cogn Psychol 29:661-668. doi:10.1002/acp.3147
Jones DP, McGraw JM (1987) Reliable and fictitious accounts of sexual abuse to children. J Interpers Violence 2:27-45. doi:10.1177 /088626087002001002

Lamb ME, Orbach Y, Sternberg KJ, Aldridge J, Pearson S, Stewart HL, Esplin PW, Bowler L (2009) Use of a structured investigative protocol enhances the quality of investigative interviews with alleged child sexual abuse in Britain. Appl Cogn Psychol 23:449-467

Lamb ME, Orbach Y, Hershkowitz I, Esplin PW, Horowitz D (2007) A structured forensic interview protocol improves the quality and informativeness of investigative interviews with children: a review of research using the NICHD investigative interview protocol. Child Abuse Negl 31:1201-1231

La Rooy D, Katz C, Malloy LC, Lamb ME (2010) Do we need to rethink guidance on repeated interviews? Psychol Public Policy Law 16: 373-392

Lawson L, Chaffin M (1992) False negatives in sexual abuse disclosure interviews: incidence and influence of caretaker's belief in abuse cases of accidental discovery and diagnosis of STD. J Interpers Violence 7:532-542

Lawson M, London K (2015) Tell me everything you discussed: children's memory for dyadic conversations after a 1-week or a 3-week delay. Behav Sci Law. doi:10.1002/bsl.2184

Leifer M, Shapiro JP, Kassem L (1993) The impact of maternal history and behaviour upon foster placement and adjustment in sexually abused girls. Child Abuse Negl 17:755-766

Lyon TD, Scurich N, Choi K, Handmaker S, Blank K (2012b) "How did you feel?": increasing child sexual abuse witnesses' production of evaluative information. Law Hum Behav 36:448-457

Lyon TD, Ahern EC, Scurich N (2012a) Interviewing children versus tossing coins: accurately assessing the diagnosticity of children's disclosures of abuse. J Child Sex Abus 21:19-44

Malloy LC, Brubacher SP, Lamb ME (2011) Expected consequences of disclosure revealed in investigative interviews with suspected victims of child sexual abuse. Appl Dev Sci 15:8-19

Malloy LC, Brubacher SP, \& Lamb ME (2013) "Because she's the one who listens": children discuss disclosure recipients in forensic interviews. Child Maltreat : $1-7$

Malloy LC, Lyon TD, Quas JA (2007) Filial dependency and recantation of child sexual abuse allegations. J Am Acad Child Adolesc Psychiatry 46:162-170

Memorandum of Good Practice (1992) London: Her Majesty's Stationary Office

Orbach Y, Hershkowitz I, Lamb ME, Sternberg KJ, Esplin PW, Horowitz D (2000) Assessing the value of structured protocols for forensic interviews of alleged child abuse victims. Child Abuse Negl 24:733-752

Powell MB, Fisher RP, Hughes-Scholes CH (2008) The effect of using trained versus untrained adult respondents in simulated practice interviews about child abuse. Child Abuse Negl 32:1007-1016. doi:10.1016/j.chiabu.2008.05.005

Reporters' Partial Trial Transcript at 32, People v. Ortega, No. PA06493701 (Cal. Super. Ct. Nov. 16-17, 2010)

Salt P, Myer M, Coleman L, Sauzier M (1990) The myth of mother as "accomplice" to child sexual abuse. In: Gomes-Schwartz B, Horowitz J, Cardelli A (eds) Child sexual abuse: the initial effects. Sage Publications, Newbury Park, pp. 109-131

Stolzenberg SN, Lyon TD (2014) Evidence summarized in attorneys' closing arguments predicts acquittals in criminal trials of child sexual abuse. Child Maltreat 19:119-129

Sternberg KJ, Lamb ME, Orbach Y, Esplin PW, Mitchell S (2001) The memorandum of good practice: theory versus application. Child Abuse Negl 25:669-981

Tremblay C, Hebert M, Piche C (1999) Coping strategies and social support as mediators of consequences in child sexual abuse victims. Child Abuse Negl 23:929-945

Zajac R, O'Neill S, Hayne H (2012) Disorder in the courtroom? Child witnesses under cross-examination. Dev Rev 32:181-204 